\title{
Convergence of the Fractional Step Lax-Friedrichs Scheme and Godunov Scheme for the Isentropic System of Gas Dynamics
}

\author{
Ding Xiaxi ${ }^{2}$, Chen Gui-Qiang ${ }^{\star 1,3}$ and Luo Peizhu ${ }^{3}$ \\ 1 Courant Institute of Mathematical Sciences, 251 Mercer Street, New York, NY 10012, USA \\ 2 Wuhan Institute of Mathematical Sciences, Academia Sinica, Wuhan 430071, China \\ 3 Institute of Systems Science, Academia Sinica, Beijing 100080, China
}

\begin{abstract}
Albstract. A convergence theorem of the fractional step Lax-Friedrichs scheme and Godunov scheme for an inhomogeneous system of isentropic gas dynamics $(1<\gamma \leqq 5 / 3)$ is established by using the framework of compensated compactness. Meanwhile, a corresponding existence theorem of global solutions with large data containing the vacuum is obtained.
\end{abstract}

\section{Introduction}

We are concerned with the following Cauchy problem (1.1)-(1.2) for an inhomogeneous system of isentropic gas dynamics:

Or

$$
\begin{gathered}
\left\{\begin{array}{l}
\rho_{t}+(\rho u)_{x}=U(\rho, u, x, t), \\
(\rho u)_{t}+\left(\rho u^{2}+p(\rho)\right)_{x}=V(\rho, u, x, t),
\end{array}\right. \\
\left.(\rho, u)\right|_{t=0}=\left(\rho_{0}(x), u_{0}(x)\right) .
\end{gathered}
$$

$$
\left\{\begin{aligned}
v_{t}+f(v)_{x} & =H(v, x, t), \\
\left.v\right|_{t=0} & =v_{0}(x, t),
\end{aligned}\right.
$$

where $v=(\rho, m)^{T}, f(v)=\left(m, m^{2} / \rho+p(\rho)\right)^{T}, H(v, x, t)=(U(\rho, m / \rho, x, t), V(\rho, m / \rho, x, t))^{T}$ and $m=\rho u, u_{0}(x)$ and $\rho_{0}(x) \geqq 0(\neq 0)$ are bounded measurable functions. For polytropic gas, $p(\rho)=k^{2} \rho^{\gamma}$, where $k$ is a constant and $\gamma>1$ is the adiabatic exponent (for usually gases $1<\gamma \leqq 5 / 3$ ).

System (1.1) is a model of gas dynamics of nonconservative form with source. For instance, $H(v, x, \hat{t})=(0, \alpha(x, t) \rho)^{T}$, where $\alpha(x, t)$ represents body force, usually gravity, acting on all the fluid in any volume. An essential feature of the system is a nonstrictly hyperbolicity, that is, a pair of wave speeds coalesce on the vacuum $\rho=0$.

\footnotetext{
¿ Partially supported by U.S. NSF Grant \# DMS-850403
} 
The homogeneous system corresponding to system (1.1) is

$$
\begin{cases}\rho_{t}+(\rho u)_{x} & =0 \\ (\rho u)_{t}+\left(\rho u^{2}+p(\rho)\right)_{x} & =0\end{cases}
$$

For the Cauchy problem of system of isentropic gas dynamics, many existence theorems of global solutions have been obtained (e.g. [1-10]). The first large data existence theorem was established by Nishida [2] for $\gamma=1$ by using Glimm method [11]. DiPerna [7] established a large data existence theorem for $\gamma=1+2 /(2 m+1)$, $m \geqq 2$ integers, by using the viscosity method and the theory of compensated compactness [12-18]. These results are both obtained provided that the initial density $\rho_{0}(x)$ is away from the vacuum for some technical reasons. A convergence theorem of the Lax-Friedrichs scheme and corresponding existence theorem of global solutions for general case $1<\gamma \leqq 5 / 3$ and large data containing the vacuum have been obtained [ $8-10]$ with the aid of an analysis of weak entropy and a study of regularity of the family of probability measures which is corresponding to the Lax-Friedrichs approximations on the basis of work of DiPerna [7].

For the general inhomogeneous cases, the term $H(v, x, t)$ does not have a preferred form, especially does not decay as $t$ goes to infinity. Thus the Duhamel principle and the energy method do not seem to be applicable here and the solution may not exist for all time.

Nevertheless, in this paper we shall use two difference schemes - the fractional step Lax-Friedrichs scheme and Godunov scheme which are generalizations of those of Lax-Friedrichs [19] and Godunov [20] - to construct approximate solutions. If the inhomogeneous terms satisfy the condition $C 1^{\circ}-C 3^{\circ}$ (Sect. 4) which especially contains cases of $(0, \alpha(x, t) \rho),(0, \alpha(x, t) \rho u),(\alpha(x, t) \rho, \alpha(x, t) \rho u)$, and $(0, \alpha(x, t) \rho u \ln (|u|+1)), \alpha(x, t) \in C\left(R \times R^{+}\right)$, we shall prove that the approximate solutions satisfy the following framework.

Theorem 1. Suppose that the inhomogeneous terms $(U, V)$ satisfy the conditions $C 1^{0}-C 2^{0}$ (Sect. 4) and the initial data $\left(\rho_{0}(x), u_{0}(x)\right)$ satisfy

$$
\left|u_{0}(x)\right| \leqq M, \quad 0 \leqq \rho_{0}(x) \leqq N, \quad \rho_{0}(x) \neq 0,
$$

and, for some constant state $(\bar{\rho}, \bar{u})$,

$$
\int_{-\infty}^{\infty}\left[\frac{1}{2} \rho_{0}(x)\left(u_{0}(x)-\bar{u}\right)^{2}+\frac{1}{\gamma(\gamma-1)}\left(\rho_{0}^{\gamma}(x)-\bar{\rho}^{\gamma}\right)-\frac{1}{\gamma-1} \bar{\rho}^{\gamma-1}\left(\rho_{0}(x)-\bar{\rho}\right)\right] d x<\infty .
$$

Then, for any $1<\gamma \leqq 2$, the difference approximate solutions $\left(\rho^{l}(x, t), m^{l}(x, t)\right)$ in the region $\Pi_{T}=\{(x, t):-\infty<x<\infty, 0 \leqq t<T\}$ satisfy

(i) There is a constant $C(T)>0$, such that

$$
0 \leqq \rho^{l}(x, t) \leqq C, \quad\left|\frac{m^{l}(x, t)}{\rho^{l}(x, t)}\right| \leqq C .
$$

(ii) The measure set

$$
\eta\left(v^{l}\right)_{t}+q\left(v^{l}\right)_{x}
$$


lies in a compact subset of $H_{\mathrm{loc}}^{-1}(\Omega)$ for all weak entropy pairs $(\eta, q)$, where $\Omega \subset \Pi_{T}$ is any bounded and open set.

From this theorem, we can obtain the following theorem by applying the results of the paper [9] and Sect. 5 of this paper.

Theorem 2. Assume that the condition $C 3^{0}$ is satisfied besides the conditions in Theorem 1. Then, for $1<\gamma \leqq 5 / 3$, there is a convergent subsequence in the approximations $\left(\rho^{l}(x, t), m^{l}(x, t)\right)$ such that

$$
\left(\rho^{l k}(x, t), m^{l k}(x, t)\right) \rightarrow(\rho(x, t), m(x, t)), \quad \text { a.e. . }
$$

Define $u(x, t)=m(x, t) / \rho(x, t)$, a.e. Then the pair of functions $(\rho(x, t), u(x, t))$ is a generalized solution of the Cauchy problem in region $\Pi_{T}$ satisfies

$$
0 \leqq \rho(x, t) \leqq C, \quad|u(x, t)| \leqq C .
$$

A crucial idea used in the limiting process in the paper [8-9] is to show that the family of Young measures which is corresponding to the approximations is a family of Dirac measures. This idea was also used by Tartar [12] and DiPerna $[6,21]$ for hyperbolic conservation laws. It is related to the theory of compensated compactness established by Murat and Tartar [10-16]. For scalar conservation law, Oleinik [24], Conway and Smoller [25], Kruzkov [26] and others proved that the approximations derived from the Lax-Friedrichs scheme or the viscosity method etc. satisfy the Helly compactness principle and obtained their convergence. For a system of hyperbolic conservation laws, however, it runs up against serious difficulties to prove that approximations, especially the Lax-Friedrichs difference approximations, satisfy this framework. This motivates people to find a new compactness framework which is satisfied by approximations (e.g. viscosity method, Lax-Friedrichs scheme and Godunov scheme, etc.) and still ensure the existence of a subsequence converging pointwise a.e. Tartar [12] first found such a compactness framework for a scalar conservation law with the aid of the idea of compensated compactness. DiPerna $[6,21]$ made a detailed analysis and established many framework theorems for hyperbolic conservation laws by using the theory of compensated compactness. In particular, DiPerna [6] obtained such a compactness framework for the viscosity method to the system of isentropic gas dynamics for $\gamma=1+2 /(2 m+1), m \geqq 2$ integers. In connection with the work of DiPerna [6], such a compactness framework has also been established [8-10] for the approximate solutions, especially Lax-Friedrichs approximations, to the system of isentropic gas dynamics for the general case $1<\gamma \leqq 5 / 3$. Theorem 2 above is obtained with the aid of the compactness framework of compensated compactness [9]. Regarding work on the framework of compensated compactness for conservation laws, we also refer the reader to Morawetz [27], Serre [28], Rascle [29], Roytburd and Slemrod [30], and Dafermos [31].

We recall that, for hyperbolic systems of conservation laws, the $L^{\infty}$ uniformly estimate of the approximations plays an important role in order to establish a convergence theorem in the method of artificial viscosity. As a general rule, one can only use the principle of invariant region or (weak) maximal principle to get the $L^{\infty}$ estimate. For the Cauchy problem (1.1)-(1.2) and $(U, V)=(0, \alpha \rho u), \alpha \leqq 0$, 
there exist bounded invariant regions [36]. For general inhomogenous terms (e.g. $(U, V)=(0, \alpha \rho u), \alpha>0)$, however, there are no bounded invariant regions in general. The difficulty can be overcome by virtue of an analysis of the solution of the nonlinear ordinary differential equation for the fractional step Lax-Friedrichs scheme and Godunov scheme.

For the study of existence of the discontinuous solutions to hyperbolic systems with inhomogeneous terms, the results which have been found are the works [32-35]. Ying Lung-an and Wang Ching-hua [33] established a global existence theorem of the Cauchy problem for an inhomogeneous system of isentropic gas dynamics $(\gamma=1)$ by using the generalized Glimm scheme. The system in the paper [33] and system (1.1) in this paper have quite different classes of inhomogeneous terms.

\section{Preliminaries}

We first introduce some basic facts before further discussion. We begin with the following facts:

A. The Homogeneous System of Gas Dynamics. Consider the system of gas dynamics

$$
\left\{\begin{array}{l}
\rho_{t}+(\rho u)_{x}=0, \\
(\rho u)_{t}+\left(\rho u^{2}+p(\rho)\right)_{x}=0,
\end{array} \quad p(\rho)=\frac{\rho^{\gamma}}{\gamma}\right.
$$

or

$$
v_{t}+f(v)_{x}=0
$$

The eigenvalues of the system are

$$
\left\{\begin{array}{l}
\lambda_{1}=u-c, \\
\lambda_{2}=u+c .
\end{array} \quad c=\sqrt{p^{\prime}(\rho)},\right.
$$

Riemann invariants are

$$
\left\{\begin{array}{l}
w=u+\frac{\rho^{\theta}}{\theta}=\frac{m}{\rho}+\frac{\rho^{\theta}}{\theta}, \\
z=u-\frac{\rho^{\theta}}{\theta}=\frac{m}{\rho}-\frac{\rho^{\theta}}{\theta} .
\end{array} \quad \theta=\frac{\gamma-1}{2}\right.
$$

1. The Elementary Wave Curves. There are two distinct types of rarefaction waves and shock waves which are denoted by $1-\mathrm{Rw}$ or 2 -Rw and 1-shock or 2-shock respectively. If a state $\left(\rho_{0}, m_{0}\right)$ or $\left(\rho_{0}, u_{0}\right)$ is given, the possible states $(\rho, m)$ or $(\rho, u)$ which can be connected to $\left(\rho_{0}, m_{0}\right)$ on the right by a Rw or shock are respectively 


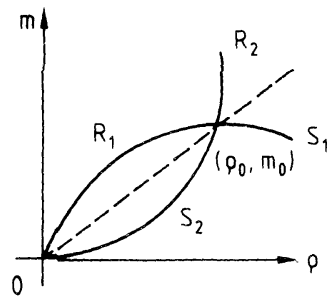

Fig. 1

$$
\begin{aligned}
& \left\{\begin{array}{l}
R_{1}(0): m-m_{0}=\frac{m_{0}}{\rho_{0}}\left(\rho-\rho_{0}\right)-\frac{\rho}{\theta}\left(\rho^{\theta}-\rho_{0}^{\theta}\right), \quad \rho<\rho_{0} \\
\text { or } \\
u-u_{0}=-\frac{1}{\theta}\left(\rho^{\theta}-\rho_{0}^{\theta}\right), \quad \rho<\rho_{0}
\end{array}\right. \\
& \left\{\begin{array}{l}
R_{2}(0): m-m_{0}=\frac{m_{0}}{\rho_{0}}\left(\rho-\rho_{0}\right)+\frac{\rho}{\theta}\left(\rho^{\theta}-\rho_{0}^{\theta}\right), \quad \rho>\rho_{0}, \\
\text { or } \\
u-u_{0}=\frac{1}{\theta}\left(\rho^{\theta}-\rho_{0}^{\theta}\right), \quad \rho>\rho_{0} .
\end{array}\right. \\
& \left\{\begin{array}{l}
S_{1}(0): m-m_{0}=\frac{m_{0}}{\rho_{0}}\left(\rho-\rho_{0}\right)-\sqrt{\frac{\rho p(\rho)-p\left(\rho_{0}\right)}{\rho_{0} \rho-\rho_{0}}}\left(\rho-\rho_{0}\right), \\
\rho>\rho_{0}>0 \\
\text { or } \quad \sqrt{\frac{1}{\rho_{0} \rho} \frac{p(\rho)-p\left(\rho_{0}\right)}{\rho-\rho_{0}}}\left(\rho-\rho_{0}\right), \quad \rho>\rho_{0}>0 . \\
u-u_{0}=-
\end{array}\right.
\end{aligned}
$$

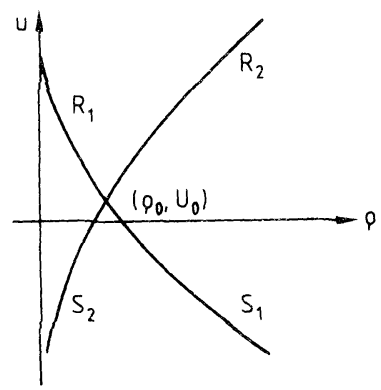

Fig. 2 


$$
\left\{\begin{array}{l}
S_{2}(0): m-m_{0}=\frac{m_{0}}{\rho_{0}}\left(\rho-\rho_{0}\right)+\sqrt{\frac{\rho}{\rho_{0}} \frac{p(\rho)-p\left(\rho_{0}\right)}{\rho-\rho_{0}}}\left(\rho-\rho_{0}\right), \quad \rho<\rho_{0}, \\
\text { or } \\
u-u_{0}=\sqrt{\frac{1}{\rho \rho_{0}} \frac{p(\rho)-p\left(\rho_{0}\right)}{\rho-\rho_{0}}}\left(\rho-\rho_{0}\right), \quad \rho<\rho_{0} .
\end{array}\right.
$$

2. The Formulae of the Centered Rarefaction $W$ ave. Along the centered rarefaction wave with central point $\left(x_{0}, t_{0}\right)$ and left state $\left(\rho_{0}, m_{0}\right)$,

$$
\left\{\begin{array}{l}
\rho(x, t)=\rho\left(\frac{x-x_{0}}{t-t_{0}}\right)=\left(\frac{\theta}{1+\theta}\right)^{1 / \theta}\left( \pm \frac{m_{0}}{\rho_{0}}+\frac{\rho_{0}^{\theta}}{\theta} \mp \frac{x-x_{0}}{t-t_{0}}\right)^{1 / \theta} \\
m(x, t)=m\left(\frac{x-x_{0}}{t-t_{0}}\right)=\left[\frac{m_{0}}{\rho_{0}} \pm \frac{\rho_{0}^{\theta}}{\theta} \mp \frac{1}{\theta}\left(\rho\left(\frac{x-x_{0}}{t-t_{0}}\right)\right)^{\theta}\right] \rho\left(\frac{x-x_{0}}{t-t_{0}}\right),
\end{array}\right.
$$

where \pm correspond to the rarefaction waves of the first and second kinds respectively. These show that, for fixed $t, \rho(x, t)$ is a monotone function of $x$ along the rarefaction waves.

3. Entropy. A pair of mappings $\eta: R^{2} \rightarrow R, q: R^{2} \rightarrow R$ is called an entropy-entropy flux pair if it satisfies an identity

$$
\nabla q=\nabla \eta \nabla f
$$

Furthermore, if $\eta(\rho, u)$ satisfies $\eta(0, u)=0$, then $\eta$ is called a weak entropy.

For example, the mechanical energy $\eta_{*}=\frac{1}{2} \rho u^{2}+\rho^{\gamma / \gamma}(\gamma-1), 1<\gamma \leqq 2$, is a strictly convex weak entropy.

One can prove that, for $0 \leqq \rho \leqq C,|u| \leqq C$,

$$
|\nabla \eta| \leqq \text { const, }
$$

and

$$
\left|\nabla^{2} \eta(r, r)\right| \leqq \text { const } \nabla^{2} \eta_{*}(r, r)
$$

where $r$ is any vector and the constant is independent of $r$.

4. The Properties of the Riemann Solution. We have the following results:

Lemma 1. Suppose that $(\rho(x, t), m(x, t))$ is a Riemann solution of system (2.1). Then the jump strength of $m(x, t)$ across an elementary wave can be dominated by that of $\rho(x, t)$ across the same elementary wave, that is,

$$
\text { across a shock wave: }\left|m_{r}-m_{l}\right| \leqq K\left|\rho_{r}-\rho_{l}\right| \text {, }
$$

on a rarefaction wave: $\left|m-m_{l}\right| \leqq K\left|\rho-\rho_{l}\right| \leqq K\left|\rho_{r}-\rho_{l}\right|,(\rho, m) \in R_{w}$,

where $K$ only depends on the upper bound of $\rho(x, t)$ and $|m(x, t) / \rho(x, t)|,\left(\rho_{l}, m_{l}\right)$ and $\left(\rho_{r}, m_{r}\right)$ denote the left and right states respectively. 
From Lemma 1, we can immediately obtain the next result.

Lemma 2. Suppose that $v(x, t)=(\rho(x, t), m(x, t))$ is a Riemann solution with central point $(0,0)$ on the rectangle: $-l<x<l, 0 \leqq t<h$. Then

$\int_{-l}^{l}|v(x, t)-v(x, h-0)|^{2} d x \leqq C \int_{-l}^{l}|\rho(x, t)-\rho(x, h-0)|^{2} d x \leqq C l \sum|\varepsilon(\rho(x, h-0))|^{2}$,

where $\varepsilon(\rho(x, h-0))$ denotes the jump strength of $\rho(x, h-0)$ across the elementary wave on $t=h, C$ only depends on the upper bound of $\rho(x, t)$ and $|m(x, t) / \rho(x, t)|$, the summation is taken over all jump strengths in $\rho(x, h-0)$ across elementary waves.

Lemma 3. If $(\rho(x, t), m(x, t))$ is a solution of Riemann problem $(1<\gamma \leqq 3)$ :

$$
\left\{\begin{array}{l}
(2.1), \quad 0<t<h, \quad-l<x<l, \\
\left.(\rho, m)\right|_{t=0}= \begin{cases}\left(\rho_{1}, m_{1}\right), & -l<x<0, \\
\left(\rho_{2}, m_{2}\right), & 0<x<l .\end{cases}
\end{array}\right.
$$

and consists of two shocks and constant states $\left(\rho_{1}, m_{1}\right),\left(\rho_{0}, m_{0}\right)$ and $\left(\rho_{0}, m_{2}\right)$ which satisfy

then we have

$$
\sigma_{2}\left(v_{0}, v_{2}\right)-\sigma_{1}\left(v_{1}, v_{0}\right) \leqq d
$$

It follows that

$$
\max \left(\rho_{1}, \rho_{2}\right) \leqq\left[\rho_{0}(d)^{2}\right]^{1 / \gamma}
$$

$$
\left|\rho_{2}-\rho_{0}\right| \leqq\left|\rho_{1}-\rho_{0}\right|+2\left(\rho_{0}(d)^{2}\right)^{1 / \gamma},
$$

where $\sigma_{i}\left(v_{l}, v_{r}\right)(i=1,2)$ denote the propagating speeds of the first and second kinds of shock waves with left state $v_{l}$ and right state $v_{r}$ respectively, the mesh length $l$ and $h$ satisfy $\max \sup \left|\lambda_{i}(\rho, m)\right|<l / 2 h \leqq K$.

$$
i=1,2
$$

Lemma 4. If $g(x)$ is a piecewise continuous function defined on some interval $[a, b]$ consisting of constant state intervals, at most two of discontinuity points and monotone continuous intervals, then

$$
\int_{a}^{b}|g(x)-\bar{g}|^{2} d x \geqq \alpha_{0} d_{0}^{3}(b-a) \sum|\varepsilon(g(x))|^{2},
$$

where

$$
\bar{g}=\frac{1}{b-a} \int_{a}^{b} g(x) d x
$$

$\varepsilon(g(x))$ denotes a jump strength of $g(x)$ across a discontinuity point or monotone continuous interval, $d_{0}$ is the infimum of ratios of the lengths of constant state intervals and $b-a$, and $\alpha_{0}>0$ only depends on $d_{0}$ and $b-a$.

It is easy to prove the fact by the analysis of various possible cases and positive definite quadratic forms.

Lemma 5. The regions $\sum=\left\{(\rho, m): w \leqq w_{0}, z \geqq z_{0}, w-z \geqq 0\right\}$ are invariant regions about Riemann problem. More precisely, if the Riemann data belong to $\sum$, the solutions of the Riemann problem belong to $\sum$ too. 
Lemma 6. If $\{(\rho(x), m(x)): a \leqq x \leqq b\} \subset \sum$, then

$$
\left(\frac{1}{b-a} \int_{a}^{b} \rho(x) d x, \frac{1}{b-a} \int_{a}^{b} m(x) d x\right) \in \sum .
$$

Lemma 7. The rate of entropy production for an arbitrary weak entropy $\eta$ is dominated by the associated rate of entropy production for $\eta_{*}$ in the sense that

$$
\left|\sigma[\eta]_{0}-[q]_{0}\right| \leqq \operatorname{const}\left\{\sigma\left[\eta_{*}\right]_{0}-\left[q_{*}\right]_{0}\right\}
$$

The proof of this fact may be found in Ref. [8].

\section{B. An Embedding Theorem}

Theorem 3. Let $\Omega \subset R^{n}$ be a bounded and open set. Then

$$
\begin{aligned}
& \text { (compact set of } \left.W^{-1, q}(\Omega)\right) \cap\left(\text { bounded set of } W^{-1, r}(\Omega)\right) \\
& \subset\left(\text { compact set of } W_{\text {loc }}^{-1,2}(\Omega)\right),
\end{aligned}
$$

where $q$ and $r$ are constants, $1<q \leqq 2<r<\infty$.

This theorem is a result of Ref. [8].

\section{Generalized Solution}

Definition. A pair of bounded measurable functions $(\rho(x, t), u(x, t))$ is called a generalized solution of the Cauchy problem (1.1)-(1.2) in the region $\Pi_{T}$, if it satisfies the following conditions:

$$
\begin{aligned}
& \iint_{0 \leqq t \leqq T}\left(\rho \phi_{t}+\rho u \phi_{x}+U(\rho, u, x, t) \phi d x d t+\int_{-\infty}^{\infty} \rho_{0}(x) \phi(x, 0) d x=0,\right. \\
& \iint_{0 \leqq t \leqq T}\left(\rho u \phi_{t}+\left(\rho u^{2}+p(\rho)\right) \phi_{x}+V(\rho, u, x, t) \phi d x d t+\int_{-\infty}^{\infty} \rho_{0}(x) u_{0}(x) \phi(x, 0) d x=0,\right.
\end{aligned}
$$

where $\phi(x, t)$ is any smooth function which has compact support in the region $\Pi_{T}$.

\section{Fractional Step Lax Friedrichs Scheme and Godunov Schemé}

In this section we shall introduce two difference schemes-the fractional step Lax-Friedrichs scheme and Godunov scheme. Meanwhile, we shall use these schemes to construct the approximate solutions $v^{l}=\left(\rho^{l}, m^{l}\right)=\left(\rho^{l}, \rho^{l} u^{l}\right)$ by means of mesh lengths $l$ and $h$ which satisfy the inequality $\max _{i=1,2}\left(\sup \left|\lambda_{i}\left(\rho^{l}, m^{l}\right)\right|\right)<$ $l / 2 h \leqq c_{0} / 2$ for any given $T>0$ and prove that $\rho^{l}(x, t) \geqq 0$, so that it is possible to construct $\left(\rho^{l}, m^{l}\right)$.

A. Fractional Step Lax-Friedrichs Scheme. For integers $n \geqq 1$, we set

$$
J_{n}=\{j: j \text { integers, } n+j=\text { even }\} .
$$

For $0 \leqq t \leqq h,(j-1) l<x<(j+1) l, j$ odd, we define 


$$
v^{l}(x, t)=v_{0}^{l}(x, t)+H\left(v_{0}^{l}(x, t), x, t\right) t
$$

where $v_{0}^{l}(x, t)=\left(\rho_{0}^{l}(x, t), m_{0}^{l}(x, t)\right)$ are the solutions of $(2.1)$ with initial data

$$
v_{0}^{l}(x)= \begin{cases}v_{0}^{l}((j-1) l), & x<j l \\ v_{0}^{l}((j+1) l), & x>j l\end{cases}
$$

where

$$
v_{0}^{l}(x)=v_{0}(x) \chi_{l}(x), \chi_{l}(x)= \begin{cases}1 & x \in\left[-\frac{1}{l}, \frac{1}{l}\right] \\ 0, & \text { otherwhere }\end{cases}
$$

From this, we define

$$
v_{j}^{1}=\frac{1}{2 l} \int_{(j-1) l}^{(j+1) l} v^{l}(x, h-0) d x .
$$

Suppose that $v^{l}(x, t)$ have been defined for $t<n h$, then define

$$
v^{l}(x, t)=v_{0}^{l}(x, t)+H\left(v_{0}^{l}(x, t), x, t\right)(t-n h),
$$

for $n h \leqq t<(n+1) h,(j-1) l<x<(j+1) l$, where $j \in J_{n}$ and $v_{0}^{l}(x, t)$ are solutions of (2.1) with initial data $\left(v_{j-1}^{n}, v_{j+1}^{n}\right)$ with respect to $j l$ at $t=n h$. Therefore, we can define the fractional step Lax-Friedrichs scheme:

$$
v_{j}^{n+1}=\frac{1}{2 l} \int_{(j-1) l}^{(j+1) l} v^{l}(x,(n+1) h-0) d x .
$$

In this way, for $n h \leqq t<(n+1) h, n \geqq 0$ integers, we have

$$
\left\{\begin{array}{l}
w^{l}(x, t)=w_{0}^{l}(x, t)+X\left(w_{0}^{l}(x, t), z_{0}^{l}(x, t), x, t, t-n h\right)(t-n h), \\
z^{l}(x, t)=z_{0}^{l}(x, t)+Y\left(w_{0}^{l}(x, t), z_{0}^{l}(x, t), x, t, t-n h\right)(t-n h),
\end{array}\right.
$$

where

$$
\left\{\begin{aligned}
X(w, z, x, t, s)= & {\left[\frac{V(\rho, u, x, t)-u U(\rho, u, x, t)}{\rho+U(\rho, u, x, t) s}\right.} \\
& \left.+\int_{0}^{1}(\rho+\tau U(\rho, u, x, t) s)^{\theta-1} d \tau U(\rho, u, x, t)\right]\left.\right|_{\substack{\rho=(\theta(w-z) / 2)^{1 / \theta} \\
u=((w+z) / 2)}} \\
Y(w, z, x, t, s)= & {\left[\frac{V(\rho, u, x, t)-u U(\rho, u, x, t)}{\rho+U(\rho, u, x, t) s}\right.} \\
& \left.-\int_{0}^{1}(\rho+\tau U(\rho, u, x, t) s)^{\theta-1} d \tau U(\rho, u, x, t)\right]\left.\right|_{\substack{\rho=(\theta(w-z) / 2)^{1 / \theta} \\
u=((w+z) / 2)}} .
\end{aligned}\right.
$$

B. Fractional Step Godunov Scheme. Similarly, for $n h \leqq t<(n+1) h, j l<x<$ $(j+1) l, j$ and $n \geqq 0$ integers, we define

$$
v^{l}(x, t)=v_{0}^{l}(x, t)+H\left(v_{0}^{l}(x, t), x, t\right)(t-n h),
$$


where $v_{0}^{l}(x, t)$ are the solutions of $(2.1)$ with initial data $\left(v_{j}^{n}, v_{j+1}^{n}\right)$ with respect to $j+\frac{1}{2}$ at $t=n h$.

From this we define the fractional step Godunov scheme:

$$
v_{j}^{n+1}=\frac{1}{l} \int_{(j-1 / 2) l}^{(j+1 / 2) l} v^{l}(x, n h-0) d x .
$$

In the same way, we can get the equalities which are similar to (3.5)-(3.6).

\section{Compactness Framework of the Approximate Solutions}

We assume that the functions $U$ and $V$ satisfy the following conditions: $C 1^{\circ}$. Both $U$ and $V$ are continuous functions,

$$
\left.\left(U, \frac{1}{\rho}(V-u U)\right)\right|_{\substack{0=0 \\ \text { or } \\ u=0}}=(0,0) .
$$

and

$$
\begin{aligned}
\mid U\left(\rho, \frac{m}{\rho}, x, t\right) & \rho \\
\mid & \leqq C_{K}, \\
\text { if }\left(\rho, \frac{m}{\rho}\right) \in S_{K} & =\left\{(\rho, m): 0 \leqq \rho \leqq K,\left|\frac{m}{\rho}\right| \leqq K\right\} .
\end{aligned}
$$

$C_{K}$ is a constant.

$C 2^{\circ}$. There exists a continuous differentiable function $F(w, z)$ and constants $h_{0}>0, M_{0} \in\left(m_{0}, \infty\right)$, where $m_{0}=\max \left(\sup _{x} w_{0}(x),-\inf _{x} z_{0}(x)\right)$,

(a) $X(w, z, x, t, s) \leqq F(w, z), Y(w, z, x, t, s) \geqq-F(w, z)$, for $w-z \geqq 0,0 \leqq t \leqq h_{0}$,

(b) $\frac{\partial F}{\partial w} \geqq 0$, for $w-z \geqq 0$, and $F(r,-r) \geqq 0$, for $r \geqq 0$.

(c) $\int_{m_{0}}^{M_{0}} \frac{d r}{F(r,-r)}>T$.

$C 3^{0} .\left|H\left(v_{2}, x, t\right)-H\left(v_{1}, x, t\right)\right| \leqq C_{K}\left|v_{2}-v_{1}\right|^{\sigma}, 0<\sigma \leqq 1$, if $v_{1}, v_{2} \in S_{K}$.

Remark 1. For $(U, V)=(0, \alpha \rho),(0, \alpha \rho u),(0, \alpha \rho u \ln (|u|+1))$ and $(\alpha \rho, \alpha \rho u)$, where $|\alpha(x, t)| \leqq \alpha_{0}<\infty$, it is easy to check that they satisfy the conditions $C 1^{0}-C 3^{0}$.

Theorem 4. Assume that the conditions $C 1^{0}-C 2^{0}$ hold and the initial data satisfy

$$
\left|u_{0}(x)\right| \leqq M, \quad 0 \leqq \rho_{0}(x) \leqq N .
$$

Then there exists a constant $h_{1}>0$ such that, when $h \leqq h_{1}$, the difference approximate solutions derived by either the fractional step Lax-Friedrichs scheme or Godunov scheme are uniformly bounded in the region $\Pi_{T}$, that is, there exists a constant $C(T)>0$ such that

$$
\left|u^{l}(x, t)\right| \leqq C, \quad 0 \leqq \rho^{l}(x, t) \leqq C, \quad(x, t) \in \Pi_{T} .
$$


Proof. First of all, we assume that the estimate (4.1) is true such that the corresponding Riemann invariant sequences satisfy

$$
w^{l}(x, t) \leqq M_{0}, \quad z^{l}(x, t) \geqq-M_{0}, \quad w^{l}(x, t)-z^{l}(x, t) \geqq 0 .
$$

Then we shall prove that there exists a constant $h_{1}>0$ indeed such that, when $h \leqq h_{1}$, the estimates with the same bound can be obtained. For concreteness, we shall only prove the result for the fractional step Lax-Friedrichs approximations provided that the conditions $C 1^{0}-C 2^{0}$ hold. The other case can be proved in the same way.

First, we shall prove that there exists a constant $h_{1}>0$, when $h \leqq h_{1}$, such that

$$
\rho^{l}(x, t) \geqq 0, \quad \text { for } \quad-\infty<x<\infty, \quad 0 \leqq t \leqq T .
$$

For $0 \leqq t \leqq h$, we obtain

$$
\begin{aligned}
\rho^{l}(x, t) & =\rho_{0}^{l}(x, t)+U\left(\rho_{0}^{l}(x, t), \frac{m_{0}^{l}(x, t)}{\rho_{0}^{l}(x, t)}, x, t\right) t \\
& =\rho_{0}^{l}(x, t)\left(\begin{array}{l}
U\left(\rho_{0}^{l}(x, t), \frac{m_{0}^{l}(x, t)}{\rho_{0}^{l}(x, t)}, x, t\right) \\
\rho_{0}^{l}(x, t)
\end{array}\right) .
\end{aligned}
$$

Observe that the condition $C 1^{0}$ and Lemma 5 , we obtain that there exists a constant $h_{2}\left(M_{0}\left(m_{0}(M, N)\right)\right)>0$, such that, when $t \leqq h_{2}$,

$$
\rho^{l}(x, t) \geqq 0 \text {. }
$$

Suppose that the above inequality holds for $t<n h$. Then for $n h \leqq t<(n+1) h$, we similarly have

$$
\rho^{l}(x, t)=\rho_{0}^{l}(x, t)\left(1+\frac{U\left(\rho_{0}^{l}(x, t), \frac{m_{0}^{l}(x, t)}{\rho_{0}^{l}(x, t)}, x, t\right)}{\rho_{0}^{l}(x, t)} t\right) \geqq 0
$$

for $h \leqq h_{2}$.

Using mathematical induction, we derive that the inequality (4.2) holds.

Moreover, for $n h \leqq t<(n+1) h, n \geqq 0$ integers, we use the condition $C 2^{0}$ to get, for $h \leqq h_{0}$,

$$
\begin{aligned}
w^{l}(x, t) & \leqq w_{0}^{l}(x, t)+F\left(w_{0}^{l}(x, t), z_{0}^{l}(x, t)\right)(t-n h) \\
& \leqq \sup _{x} w_{0}^{l}(x, n h+0)+F\left(\sup _{x} w_{0}^{l}(x, n h+0), \inf _{x} z_{0}^{l}(x, n h+0)\right)(t-n h), \\
z^{l}(x, t) & \geqq z_{0}^{l}(x, t)-F\left(w_{0}^{l}(x, t), z_{0}^{l}(x, t)\right)(t-n h) \\
& \geqq \inf _{x} z_{0}^{l}(x, n h+0)-F\left(\sup _{x} w_{0}^{l}(x, n h+0), \inf _{x} z_{0}^{l}(x, n h+0)\right)(t-n h) .
\end{aligned}
$$

In particular, we obtain

$$
w^{l}(x,(n+1) h-0) \leqq \sup _{x} w_{0}^{l}(x, n h+0)
$$




$$
\begin{aligned}
& +F\left(\sup _{x} w_{0}^{l}(x, n h+0), \inf _{x} z_{0}^{l}(x, n h+0)\right) h, \\
z^{l}(x,(n+1) h-0) \geqq \inf _{x}^{l}(x, n h+0) & \\
& -F\left(\sup _{x} w_{0}^{l}(x, n h+0), \inf _{x} z_{0}^{l}(x, n h+0)\right) h .
\end{aligned}
$$

Set $M_{n}=\max \left(\sup _{x} w_{0}^{l}(x, n h+0),-\inf _{x} z_{0}^{l}(x, n h+0)\right)$. Then we have

$$
\operatorname{Max}\left(\sup _{x} w^{l}(x,(n+1) h-0),-\inf _{x} z^{l}(x,(n+1) h-0)\right) \leqq M_{n}+F\left(M_{n},-M_{n}\right) h .
$$

It follows that

that is,

$$
M_{n+1} \leqq M_{n}+F\left(M_{n},-M_{n}\right) h,
$$

$$
\frac{M_{n+1}-M_{n}}{h} \leqq F\left(M_{n},-M_{n}\right) \text {. }
$$

Consider the corresponding ordinary differential equation.

$$
\left\{\begin{array}{l}
\frac{d r}{d t}=F(r,-r) \\
r(0)=m_{0} \equiv \max \left(\sup _{x} w_{0}(x),-\inf _{x} z_{0}(x)\right) .
\end{array}\right.
$$

It follows that

$$
\int_{m_{0}}^{r(t)} \frac{d r}{F(r,-r)}=t
$$

Then, from the condition $C 2^{\circ}(\mathrm{c})$, there exists a constant $M_{0}(T)<\infty$ such that

Meanwhile

$$
m_{0} \leqq r(t) \leqq M_{0}, \quad \text { for } \quad 0 \leqq t \leqq T
$$

$$
\frac{d^{2} r(t)}{d t^{2}}=\left(F_{w}(r(t),-r(t))-F_{z}(r(t),-r(t))\right) F(r(t),-r(t)) \geqq 0 .
$$

This shows that the integral curve $r=r(t)$ is convex.

It follows from (4.2)-(4.5) that

$$
M_{n} \leqq r(n h) \leqq M_{0} .
$$

We derive from (4.2) and (4.7) that

$$
w^{l}(x, t) \leqq M_{0},-z^{l}(x, t) \leqq M_{0} \quad \text { and } \quad w^{l}(x, t)-z^{l}(x, t) \geqq 0,
$$

that is, for $h \leqq h_{1}=\min \left(h_{0}, h_{2}\right)$, there is a constant $C(T)$ such that

$$
\left|u^{l}(x, t)\right|=\left|\frac{m^{l}(x, t)}{\rho^{l}(x, t)}\right| \leqq C, \quad 0 \leqq \rho^{l}(x, t) \leqq C .
$$


The proof of the other case can be similarly obtained.

Theorem 5. Assume that the conditions in Theorem 4 and (1.5) are satisfied. Then, for $1<\gamma \leqq 2$, the measure set

$$
\eta\left(v^{l}\right)_{t}+q\left(v^{l}\right)_{x}
$$

lies in a compact subset of $H_{\mathrm{loc}}^{-1}(\Omega)$ for all weak pair $(\eta, q)$, where $\Omega \subset \Pi_{T}$ is any bounded and open set.

Proof. For simplicity in printing we shall drop the index of the approximate solutions $v^{l}(x, t)$ and $v_{0}^{l}(x, t)$ in the process of the proof and only prove the result for the fractional step Lax-Friedrichs approximations.

Step 1. The entropy equality can be written in the form

$$
\iint_{0 \leqq t \leqq T=m h}\left(\eta(v) \phi_{t}+q(v) \phi_{x}\right) d x d t=M(\phi)+N(\phi)+L(\phi)+\sum(\phi),
$$

where

$$
\begin{gathered}
M(\phi)=\int \phi(x, T) \eta\left(v_{0}(x, T)\right) d x-\int \phi(x, 0) \eta\left(v_{0}(x, 0)\right) d x, \\
N(\phi)=\iint\left[\left(\eta(v)-\eta\left(v_{0}\right)\right) \phi_{1}+\left(q(v)-q\left(v_{0}\right)\right) \phi_{x}\right] d x d t \\
L(\phi)=\sum_{j, n} \int_{(j-1) l}^{(j+1) l}\left[\left(\eta\left(v_{0-}^{n}\right)-\eta\left(v_{0 j}^{n}\right)\right] \phi(x, n h) d x \equiv L_{1}(\phi)+L_{2}(\phi)+L_{3}(\phi),\right. \\
L_{1}(\phi)=\sum_{j, n} \phi_{j}^{n} \int_{(j-1) l}^{(j+1) l}\left(\eta\left(v_{-}^{n}\right)-\eta\left(v_{0 j}^{n}\right)\right) d x \\
L_{2}(\phi)=\sum_{j, n}^{(j+1) l} \int_{(j-1) l}^{(j+1) l}\left(\eta\left(v_{0-}^{n}\right)-\eta\left(v_{-}^{n}\right)\right) \phi(x, n h) d x \\
L_{3}(\phi)=\sum_{j, n} \int_{(j-1) l}^{T}\left(\eta\left(v_{-}^{n}\right)-\eta\left(v_{0 j}^{n}\right)\right)\left(\phi-\phi_{j}^{n}\right) d x \\
\sum(\phi)=\int_{0}^{T} \sum\left\{\sigma[\eta]_{0}-[q]_{0}\right\} \phi(x(t), t) d t
\end{gathered}
$$

where $v_{-}^{n}=v(x, n h-0), \phi_{j}^{n}=\phi(j l, n h)$, the summation is taken over all shock waves in $v$ at a fixed time $t, \sigma$ is the propagating speed of the shock wave.

Let $S=(x(t), t)$ denote a shock wave in $v_{0}(x, t),[\eta]_{0}$ and $[q]_{0}$ denote the jump of $\eta\left(v_{0}(x, t)\right)$ and $q\left(v_{0}(x, t)\right)$ across $S$ from left to right respectively, namely,

$$
\left\{\begin{array}{l}
{[\eta]_{0}=\eta\left\{v_{0}(x(t)+0, t)\right\}-\eta\left\{v_{0}(x(t)-0, t)\right\},} \\
{[q]_{0}=q\left\{v_{0}(x(t)+0, t)\right\}-q\left\{v_{0}(x(t)-0, t)\right\} .}
\end{array}\right.
$$

Step 2. Without loss of generality we suppose

$$
\int_{-\infty}^{\infty} \eta_{*}\left(\rho_{0}(x), u_{0}(x)\right) d x<\infty
$$

otherwise one need only introduce a normalized entropy pair 


$$
\left\{\begin{array}{l}
\tilde{\eta}_{*}=\eta_{*}(v)-\eta_{*}(\bar{v})-\nabla \eta_{*}(\bar{v})(v-\bar{v}) \\
\tilde{q}_{*}=q_{*}(v)-q_{*}(\bar{v})-\nabla \eta_{*}(\bar{v})(f(v)-f(\bar{v}))
\end{array}\right.
$$

and then repeating the argument below.

Observe that $(\rho, u)$ have compact support in the region $\Pi_{T}$ and $\left.(U, V)\right|_{\substack{\rho=0 \\ \text { or }}}=(0,0)$, we may substitute

$$
\eta=\eta_{*}=\frac{1}{2} \rho u^{2}+\frac{1}{\gamma(\gamma-1)} \rho^{\gamma}, \quad q=q_{*}=\frac{1}{2} \rho u^{3}+\frac{1}{\gamma-1} \rho^{\gamma} u \quad \text { and } \quad \phi=1
$$

in the equality (4.7). Thus

$$
\sum_{n=1}^{m} \int\left[\eta_{*}^{n}\right]_{0} d x+\int_{0}^{T} \sum\left\{\sigma\left[\eta_{*}\right]_{0}-\left[q_{*}\right]_{0}\right\} d t \leqq C
$$

while

$$
\begin{aligned}
\sum_{n=1}^{m} \int\left[\eta_{*}^{n}\right]_{0} d x= & \sum_{j, n} \int_{(j-1) l}^{(j+1) l}\left[\eta_{*}\left(v_{0-}^{n}\right)-\eta_{*}\left(v_{0 j}^{n}\right)\right] d x \\
= & \sum_{j, n} \int_{(j-1) l}^{(j+1) l} \int_{0}^{1}(1-\theta) \nabla^{2} \eta_{*}\left(v_{0 j}^{n}+\theta\left(v_{-}^{n}-v_{0 j}^{n}\right)\right) d \theta\left(v_{-}^{n}-v_{0 j}^{n}\right)^{2} d x \\
& -\sum_{j, n}^{(j+1) l} \int_{(j-1) l}^{1} \int_{0}^{1} \nabla \eta_{*}\left(v_{0-}^{n}+\theta\left(v_{-}^{n}-v_{0-}^{n}\right)\right) d \theta\left(v_{-}^{n}-v_{0-}^{n}\right) d x .
\end{aligned}
$$

But

$$
\begin{aligned}
& \left|\sum_{j, n} \int_{-1, l) l}^{(j+1) l}\left(\int_{0}^{1} \nabla \eta_{*}\left(v_{0-}^{n}+\theta\left(v_{-}^{n}-v_{0-}^{n}\right)\right) d \theta\right)\left(v_{-}^{n}-v_{0-}^{n}\right) d x\right| \\
& \quad \leqq \sum_{j, n} \int_{-}^{(j+1) l} \int_{0}^{1}\left|\nabla \eta_{*}\left(v_{0-}^{n}+\theta\left(v_{-}^{n}-v_{0-}^{n}\right)\right)\right| d \theta\left|H\left(v_{0}^{n}, x, t\right)\right| d x \cdot h \leqq C .
\end{aligned}
$$

Notice that the entropy inequality $\sigma\left[\eta_{*}\right]_{0}-\left[q_{*}\right]_{0} \geqq 0$ is satisfied $[10]$ across the shock waves and $\eta_{*}$ is a convex entropy. We have from (4.15)

$$
\begin{gathered}
\int_{0}^{T} \sum\left\{\sigma\left[\eta_{*}\right]_{0}-\left[q_{*}\right]_{0}\right\} d t \leqq C, \\
\sum_{j, n} \int_{(j-1) l}^{(j+1) l}\left[\int_{0}^{1}(1-\theta) \nabla^{2} \eta_{*}\left(v_{0 j}^{n}+\theta\left(v_{-}^{n}-v_{0 j}^{n}\right) d \theta\right]\left(v_{-}^{n}-v_{0 j}^{n}\right)^{2} d x \leqq C .\right.
\end{gathered}
$$

In particular, since $\nabla^{2} \eta_{*}(r, r) \geqq c_{0}(r, r), c_{0}>0$ constant, we get

$$
\sum_{j, n} \int_{(j-1) l}^{(j+1) l}\left|v_{-}^{n}-v_{0 j}^{n}\right|^{2} d x \leqq C \text {. }
$$

It follows that

$$
\sum_{\substack{j, n \\|j l| \leqq L}}^{(j+1) l} \int_{(j-1) l}^{\left(j_{0}\right.}\left|v_{0-}^{n}-v_{0 j}^{n}\right|^{2} d x \leqq C(L)
$$

Step 3. For any bounded set $\Omega \subset \Pi_{T}$ and weak entropy pair $(\eta, q)$, we derive from 
(4.8), (4.12)-(4.13), (4.15), (4.16)-(4.17) and Lemma 7 that

$$
\begin{aligned}
|M(\phi)| & \leqq C\|\phi\|_{C_{0}(\Omega)}, \\
\left|\sum(\phi)\right| & \leqq C\|\phi\|_{C_{0}(\Omega)} \int_{0}^{T} \sum\left(\sigma\left[\eta_{*}\right]-\left[q_{*}\right]\right) d x \leqq C\|\phi\|_{C_{0}(\Omega)}, \\
\left|L_{1}(\phi)\right| & \leqq\left|\sum_{j, n} \phi_{j}^{n} \int_{(j-1) l}^{(j+1) l}\left(\eta\left(v_{-}^{n}\right)-\eta\left(v_{0 j}^{n}\right)\right) d x\right| \\
& \leqq\|\phi\|_{C_{0}(\Omega)} \sum_{j, n} \int_{(j-1) l}^{(j+1) l} \int_{0}^{1}(1-\theta)\left|\nabla^{2} \eta\left(v_{0 j}^{n}+\theta\left(v_{-}^{n}-v_{0 j}^{n}\right)\right)\left(v_{-}^{n}-v_{0 j}^{n}\right)^{2}\right| d \theta d x \\
& \leqq C\|\phi\|_{C_{0}(\Omega)} \sum_{j, n}^{(j+1) l} \int_{j-1) l}^{1}(1-\theta) \nabla^{2} \eta_{*}\left(v_{0 j}^{n}+\theta\left(v_{-}^{n}-v_{0 j}^{n}\right)\right)\left(v_{--}^{n}-v_{0 j}^{n}\right)^{2} d \theta d x \\
& \leqq C\|\phi\|_{C_{0}(\Omega)}, \\
\left|L_{2}(\phi)\right| & =\left|\sum_{j, n} \int_{(j-1) l}^{(j+1) l}\left[\eta\left(v_{0-}^{n}\right)-\eta\left(v_{-}^{n}\right)\right] \phi(x, n h) d x\right| \\
& \leqq C l \sum_{j, n} \int_{(j-1) l}^{(j+1) l}\left|H\left(v_{0}(x, n h-0), x, t\right)\right||\phi(x, n h)| d x \\
& \leqq C\|\phi\|_{C_{0}(\Omega)},
\end{aligned}
$$

where the constant $C$ only depends on the support of $\phi$. Hence

$$
\left|\left(M+L_{1}+L_{2}+\sum\right)(\phi)\right| \leqq C\|\phi\|_{C_{0}},
$$

that is

$$
\left\|M+L_{1}+L_{2}+\sum\right\|_{C_{0}^{*}} \leqq C
$$

Therefore

$$
M+L_{1}+L_{2}+\sum \text { is compact in } W^{-1, q_{1}}(\Omega),
$$

where $1<q_{1}<n /(n-1)$.

Furthermore, for any $\phi \in C_{0}^{\beta}(\Omega), \frac{1}{2}<\beta<1$, we have

$$
\begin{aligned}
\left|L_{3}(\phi)\right| & \leqq \sum_{j, n} \int_{(j-1) l}^{(j+1) l}\left|\phi(x, n h)-\phi_{j}^{n}\right|\left|\eta\left(v_{-}^{n}\right)-\eta\left(v_{0 j}^{n}\right)\right| d x \\
& \leqq l^{\beta}\|\phi\|_{C_{0}^{\beta}} \sum_{n}\left(\sum_{j} \int_{(j-1) l}^{(j+1) l}\left|\eta\left(v_{-}^{n}\right)-\eta\left(v_{0 j}^{n}\right)\right|^{2} d x\right)^{1 / 2} \\
& \leqq l^{\beta-1 / 2}\|\nabla \eta\|_{L^{\infty}}\|\phi\|_{C_{0}^{\beta}}\left(\sum_{j, n} \int_{(j-1) l}^{(j+1) l}\left|v_{-}^{n}-v_{0 j}^{n}\right|^{2} d x\right)^{1 / 2} \\
& \leqq 2 C l^{\beta-1 / 2}\|\phi\|_{C_{0}^{\beta}(\Omega)} .
\end{aligned}
$$

Using the Sobolev theorem: $W_{0}^{1, p}(\Omega) \subset C_{0}^{\beta}(\Omega), 0<\beta<1-n / p$, we have

$$
\left|L_{3}(\phi)\right| \leqq C l^{\beta-1 / 2}\|\phi\|_{w_{0}^{1, p}(\Omega)}, \quad p>\frac{n}{1-\beta},
$$


that is

$$
\left\|L_{3}\right\|_{W^{-1, q_{2}}}(\Omega) \leqq C l^{\beta-1 / 2} \rightarrow 0, \quad(l \rightarrow 0), \quad 1<q_{2}<\frac{n}{n-1+\beta} .
$$

It follows from (4.19)-(4.20) that

$$
M+L+\sum \text { is compact in } W^{-1, q_{0}}(\Omega),
$$

where $1<q_{0}=\min \left(q_{1}, q_{2}\right)<n /(n-1+\beta)$.

Observe that $0 \leqq \rho \leqq C$ and $|u| \leqq C$. We have

that is

$$
\eta(v)_{t}+q(v)_{x}-N \text { is a bounded set of } W^{-1, r}(\Omega)(r>1),
$$

$$
M+L+\sum \text { is a bounded set of } W^{-1, r}(\Omega)(r>1) .
$$

We derive from (4.21)--(4.22) and Theorem 3 that

$$
M+L+\sum \text { is compact in } H_{\mathrm{loc}}^{-1}(\Omega) \text {. }
$$

Furthermore, for any $\phi \in C_{0}^{\infty}(\Omega)$, we have

$$
|N(\phi)| \leqq C l \int_{\operatorname{supp} \phi}\left(\left|\phi_{t}\right|+\left|\phi_{x}\right|\right) d x d t \leqq C l\|\phi\|_{H_{0}^{1}(\Omega)} .
$$

Notice that $C_{0}^{\infty}(\Omega)$ is dense in $H_{0}^{1}(\Omega)$, it follows that

that is

$$
\|N\|_{H_{\mathrm{loc}}^{-1}(\Omega)} \leqq C l \rightarrow 0, \quad(l \rightarrow 0)
$$

$$
N \text { is compact in } H_{\mathrm{loc}}^{-1}(\Omega) \text {. }
$$

So far, we have obtained from (4.8), (4.23)-(4.24)

$$
\eta\left(v^{l}\right)_{t}+q\left(v^{l}\right)_{x} \text { is compact in } H_{\mathrm{loc}}^{-1}(\Omega) .
$$

This completes the proof of the theorem.

From the results of Theorem 4 and Theorem 5, we can obtain Theorem 2 (Sect. 1), namely, the compactness framework theorem, of the difference approximate solutions.

\section{Existence Problem}

In this section we shall discuss the existence problem about the generalized solution of the Cauchy problem (1.1)-(1.2). We have the following theorem.

Theorem 6. Assume that the inhomogeneous terms satisfy the condition $C 3^{\circ}$, and the approximate solutions $v^{l}(x, t)=\left(\rho^{l}(x, t), m^{l}(x, t)\right)$ derived by either the fractional step Lax-Friedrichs scheme or Godunov scheme satisfy:

(i) There is a constant $C_{1}(T)>0$ and $C_{2}(L)>0$ such that

$$
\begin{aligned}
0 \leqq & \rho^{l}(x, t) \leqq C_{1}, \quad\left|u^{l}(x, t)\right| \leqq C_{1}, \quad(x, t) \in \Pi_{T} . \\
& \sum_{j, n} \int_{(j-1) l}^{(j+1) l}\left|v_{0-}^{l n}-v_{0 j}^{l n}\right|^{2} d x \leqq C_{2},
\end{aligned}
$$


where

$$
v_{0-}^{l n}=v_{0}^{l}(x, n h-0) .
$$

(ii) There is a convergent subsequence (still denoted by $\left.v^{l}(x, t)=\left(\rho^{l}(x, t), m^{l}(x, t)\right)\right)$ such that

$$
\left(\rho^{l}(x, t), m^{l}(x, t)\right) \rightarrow(\rho(x, t), m(x, t)), \quad \text { a.e. . }
$$

Define $u(x, t)=m(x, t) / \rho(x, t)$, a.e. . Then the pair of functions $(\rho(x, t), u(x, t))$ is $a$ generalized solution of the Cauchy problem (1.1)-(1.2) in the region $\Pi_{T}$ and satisfies

$$
0 \leqq \rho(x, t) \leqq C, \quad|u(x, t)| \leqq C, \quad \text { a.e. }
$$

Proof. For any function $\phi(x, t) \in C_{0}^{\infty}\left(\Pi_{T}\right)$, we have

$$
\begin{aligned}
& \iint_{I_{T}}\left(\phi_{t} v^{l}(x, t)+\phi_{x} f\left(v^{l}(x, t)\right)+\phi H\left(v^{l}(x, t), x, t\right)\right) d x d t+\int \phi v^{l}(x, 0) d x \\
& =\iint_{I_{T}}\left(\phi_{t} v_{0}^{l}(x, t)+\phi_{x} f\left(v_{0}^{l}(x, t)\right)+\phi H\left(v_{0}^{l}(x, t), x, t\right)\right) d x d t+\int \phi v_{0}^{l}(x, t) d x \\
& \quad+\sum_{n=0}^{m-1} \int_{n h \leqq t<(n+1) h}\left[\left(\phi_{t}+\phi_{x} \cdot \int_{0}^{1} f^{\prime}\left(v_{0}^{l}+\theta\left(v^{l}-v_{0}^{l}\right)\right) d \theta\right) H\left(v_{0}^{l}, x, t\right)(t-n h)\right. \\
& \left.\quad+\phi\left(H\left(v^{l}, x, t\right)-H\left(v_{0}^{l}, x, t\right)\right)\right] d x d t \equiv I_{1}+I_{2} .
\end{aligned}
$$

Remark that $\left|v^{l}-v_{0}^{l}\right| \leqq H\left(v_{0}^{l}, x, t\right) h \leqq C l$ and uniformed bounded of $v^{l}$. We have

$$
\begin{aligned}
\left|I_{2}\right| \leqq & h \iint\left|\phi_{t}+\phi_{x} \int_{0}^{1} f^{\prime}\left(v_{0}^{l}+\theta\left(v^{l}-v_{0}^{l}\right)\right) d \theta\right|\left|H\left(v_{0}^{l}, x, t\right)\right| d x d t \\
& +\iint|\phi|\left|H\left(v^{l}, x, t\right)-H\left(v_{0}^{l}, x, t\right)\right| d x d t \\
\leqq & C l+C \int_{\operatorname{supp} \phi}\left|H\left(v^{l}, x, t\right)-H\left(v_{0}^{l}, x, t\right)\right| d x d t \rightarrow 0, \quad(l \rightarrow 0) .
\end{aligned}
$$

Furthermore,

$$
I_{1}=\sum_{n=1}^{m-1} \int \phi(x, n h)\left[v_{0}^{l n}\right] d x+\iint \phi H\left(v_{0}^{l}, x, t\right) d x d t \equiv I_{11}+I_{12},
$$

where

$$
\begin{aligned}
& \left|I_{11}\right|=\left|\sum_{j, n} \int_{(j-1) l}^{(j+1) l}\left(\phi-\phi_{j}^{n}\right)\left(v_{0-}^{l n}-v_{0 j}^{l n}\right) d x\right| \\
& \leqq C l^{1 / 2}\|\phi\|_{C_{0}^{1}}\left\{\sum_{j, n} \int_{(j, l \mid \leqq L}^{(j+1) l}\left|v_{0-1) l}^{l n}-v_{0 j}^{l n}\right|^{2} d x\right\}^{1 / 2} \\
& \leqq C l^{1 / 2} \rightarrow 0, \quad(l \rightarrow 0), \\
& \left|I_{12}\right|=\mid \sum_{j, n} \phi_{j}^{n} \int_{(j-1) l}^{(j+1) l}\left[v_{0}^{l}(x, n h-0)-\frac{1}{2 l} \int_{(j-1) l}^{(j+1) l} v_{0}^{l}(x, n h-0) d x\right. \\
& \left.-\frac{h}{2 l} \int_{(j-1) l}^{(j+1) l} H\left(v_{0}^{l}(x, n h-0), x, n h-0\right) d x\right] d x+\iint \phi H\left(v_{0}^{l}, x, t\right) d x d t
\end{aligned}
$$




$$
\begin{aligned}
= & \left|-h \sum_{j, n} \phi_{j}^{n} \int_{(j-1) l}^{(j+1) l} H\left(v_{0}^{l}(x, n h-0), x, n h-0\right) d x+\iint \phi H\left(v_{0}^{l}(x, t), x, t\right) d x d t\right| \\
= & \mid \sum_{j, n} \int_{(n-1) h}^{n h} d t \int_{(j-1) l}^{(j+1) l}\left[\phi(x, t) H\left(v_{0}^{l}(x, t), x, t\right)\right. \\
& \left.-\phi(j l, n h) H\left(v_{0}^{l}(x, n h-0), x, n h-0\right)\right] d x \mid \leqq J_{1}+J_{2} \\
& J_{1}=\left|\sum_{j, n} \int_{(n-1) h}^{n h} d t \int_{(j-1) l}^{(j+1) l}[\phi(x, t)-\phi(j l, n h)] H\left(v_{0}^{l}(x, t), x, t\right) d x\right| \\
& \leqq C l\|\phi\|_{C^{1}} \rightarrow 0, \quad(l \rightarrow 0)
\end{aligned}
$$

From Lemma 2 (Sect. 2), we have

$$
\begin{aligned}
J_{2} & =\left|\sum_{j, n} \int_{(n-1) h}^{n h} \int_{(j-1) l}^{(j+1) l} \phi(j l, n h)\left[H\left(v_{0}^{l}(x, t), x, t\right)-H\left(v_{0}^{l}(x, n h-0), x, n h-0\right)\right] d x\right| \\
& \leqq C\left(\sum_{j, n} \int_{(n-1) h}^{n h} d t \int_{(j-1) l}^{(j+1) l}|\phi(j l, n h)|\left|v_{0}^{l}(x, t)-v_{0}^{l}(x, n h-0)\right|^{\sigma} d x+o(1)\right) \\
& \leqq C\left(\left(\sum_{n} \int_{(n-1) h}^{n h} d t \int_{-L}^{L}\left|v_{0}^{l}(x, t)-v_{0}^{l}(x, n h-0)\right|^{2} d x\right)^{\sigma / 2}+o(1)\right) \\
& \leqq C\left(\left(\sum_{n} \int_{(n-1) h}^{n h} d t \int_{-L}^{L}\left|\rho_{0}^{l}(x, t)-\rho_{0}^{l}(x, n h-0)\right|^{2} d x\right)^{\sigma / 2}+o(1)\right) \\
& \leqq C\left(\Delta_{l}^{\sigma / 2}+o(1)\right) .
\end{aligned}
$$

Suppose that $v^{l}=\left(\rho^{l}, m^{l}\right)=\left(\rho^{l}, \rho^{l} u^{l}\right)$ are Riemann solutions of the initial data

$$
\left.(\rho, m)\right|_{t=(n-1) h}= \begin{cases}\left(\rho_{1}, m_{1}\right) & x<j l, \\ \left(\rho_{2}, m_{2}\right) & x>j l,\end{cases}
$$

on the rectangle $\{(n-1) h \leqq t<n h,(j-1) l<x<(j+1) l\}$, and the intermediate constant state (provided that it exists) is $\left(\rho_{0}, m_{0}\right)$.

(i) If $v^{l}(x, t)$ consist of either constant states, 1-shock and 2-shock, and the ratios of lengths of the interval of intermediate constant state and $l$ are smaller than $\delta$; or constant states, 1-Rw and 2-shock, and the intermediate constant state $\rho_{0} \leqq \delta^{1 / \theta}$, then

$$
\int_{(j-1) l}^{(j+1) l}\left|\rho_{0}^{l}(x, t)-\rho_{0}^{l}(x, n h-0)\right|^{2} d x \leqq C \delta l, \quad(n-1) h \leqq t<n h .
$$

(ii) In other situations, we define $\bar{\rho}^{l}(x, t)$ as follows:

a. For $2-R w$ of $v^{l}(x, t)$ with the left state $v_{-}$, we define

$$
\bar{\rho}(x, t)= \begin{cases}\bar{\rho}_{-}, & x-j l \leqq \lambda_{i}\left(\bar{\rho}_{-}, \bar{\rho}_{-} u^{l}(x, t)\right)(t-n h), \\ \rho^{l}(x, t), & x-j l>\lambda_{i}\left(\bar{\rho}_{-}, \bar{\rho}_{-} u^{l}(x, t)\right)(t-n h) .\end{cases}
$$

b. For $1-R w$ and $i$-shock $(i=1,2)$ of $v^{l}(x, t)$, we define 


$$
\bar{\rho}^{l}(x, t)=\rho^{l}(x, t),
$$

where $\bar{\rho}_{ \pm}=\max \left(\rho_{ \pm}, \delta^{1 / \theta}\right)$, for sufficiently small $\delta>0$. Then, for this case (ii), the ratios of lengths of the interval of intermediate constant state $\bar{\rho}_{0} \geqq \delta^{1 / \theta}$ (provided that it exists) and $l$ of $\bar{\rho}^{l}(x, t)$ are all bigger than $\delta$ and

$$
\begin{aligned}
& \int_{(j-1) l}^{(j+1) l}\left(\bar{\rho}^{l}(x, t)-\left.\rho^{l}(x, t)\right|^{2}+\left|\bar{\rho}_{0 j}^{n}-\rho_{0 j}^{n}\right|^{2}\right) d x \\
& \quad \leqq C \delta^{(\gamma+3) /(\gamma-1)} l,(n-1) h \leqq t<n h .
\end{aligned}
$$

Using Lemma 4, we have

$$
\int_{(j-1) l}^{(j+1) l}\left|\bar{\rho}_{0-}^{n}-\bar{\rho}_{0 j}^{n}\right|^{2} d x \geqq \alpha_{0} \delta^{3} l\left|\varepsilon\left(\bar{\rho}_{0}^{l}(x, n h-0)\right)\right|^{2} .
$$

Now we divide $\Delta_{l}$ into two parts:

$$
\begin{aligned}
\Delta_{l}= & \sum_{\substack{j, n \\
|j l| \leqq L}}^{*} \int_{(n-1) h}^{n h} d t \int_{(j-1) l}^{(j+1) l}\left|\rho^{l}(x, t)-\rho^{l}(x, n h-0)\right|^{2} d x \\
& +\sum_{j, n}^{* *} \int_{(j, \mid \leqq L}^{n h} \dot{d} d t \int_{(j-1) l}^{(j+1) l}\left|\rho^{l}(x, t)-\rho^{l}(x, n h-0)\right|^{2} d x,
\end{aligned}
$$

where the summations $\sum^{*}$ and $\sum^{* *}$ are respectively take over the rectangles where the case (i) and case (ii) occur.

Therefore, we obtain for (5.7), (5.11)-(5.12),

$$
\begin{aligned}
\Delta_{l} \leqq & 3 \sum_{j, n}^{* *} \int_{(j l \mid \leqq L}^{n h} d t \int_{(j-1) l}^{(j+1) l}\left(\left|\bar{\rho}_{0}^{l}(x, t)-\bar{\rho}_{0}^{l}(x, n h-0)\right|^{2}\right. \\
& \left.+\left|\rho^{l}(x, t)-\bar{\rho}_{0}^{l}(x, t)\right|^{2}+\left|\bar{\rho}_{0}^{l}(x, n h-0)-\rho_{0}^{l}(x, n h-0)\right|^{2}\right) d x+C \delta \\
\leqq & C\left(l^{2} \sum_{j, n}^{* *}\left|\varepsilon\left(\bar{\rho}_{0}^{l}(x, n h-0)\right)\right|^{2}+\delta\right) \\
\leqq & C\left(\delta^{-3} l \sum_{j, n}^{* *} \int_{(j-1)}^{(j+1) !}\left|\bar{\rho}_{0}^{n}-\bar{\rho}_{0 j}^{n}\right|^{2} d x+\delta\right) \\
\leqq & C\left(\delta^{-3} l \sum_{j, n} \int_{(j-1) l}^{(j+1) l}\left|\rho_{0}^{n}-\rho_{0 j}^{n}\right|^{2} d x+\delta^{2(3-\gamma) /(\gamma-1)}+\delta\right) \\
\leqq & C\left(\delta^{-3} l+\delta^{2(3-\gamma) /(\gamma-1)}+\delta\right) .
\end{aligned}
$$

This shows that for sufficiently small and arbitrary constant $\delta>0$,

$$
\lim _{l \rightarrow 0} \Delta_{l} \leqq C\left(\delta^{2(3-\gamma) /(\gamma-1)}+\delta\right) .
$$

It follows that 
that is,

$$
J_{2} \rightarrow 0, \quad(l \rightarrow 0),
$$

Observe that

$$
I_{1}+I_{2} \rightarrow 0, \quad(l \rightarrow 0)
$$

$$
\left(\rho^{l}, m^{l}\right) \rightarrow(\rho, m), \quad \text { a.e. . }
$$

We obtain

$$
0 \leqq \rho \leqq C, \quad|u|=\frac{|m|}{\rho} \leqq C, \quad \text { a.e. }
$$

Using the control convergence theorem we derive from (5.1) that

$$
\begin{gathered}
\iint_{I_{I}}\left[\phi_{t} \cdot \rho(x, t)+\phi_{x} \cdot m(x, t)+\phi \cdot U\left(\rho(x, t), \frac{m(x, t)}{\rho(x, t)}, x, t\right)\right] d x d t \\
+\int_{-\infty}^{\infty} \phi(x, 0) \rho_{0}(x) d x=0, \\
\iint_{I_{T}}\left[\phi_{t} \cdot m(x, t)+\phi_{x} \cdot\left(\frac{m(x, t)^{2}}{\rho(x, t)}+\rho(\rho(x, t))\right)+\phi \cdot V\left(\rho(x, t), \frac{m(x, t)}{\rho(x, t)}, x, t\right)\right] d x d t \\
+\int_{-\infty}^{\infty} \phi(x, 0) m_{0}(x) d x=0 .
\end{gathered}
$$

Define $u(x, t)=m(x, t) / \rho(x, t)$, a.e. Then we obtain

$$
\begin{gathered}
\iint_{\Pi_{T}}\left[\phi_{t} \cdot \rho(x, t)+\phi_{x} \cdot(\rho u)(x, t)+\phi \cdot U(\rho(x, t), u(x, t), x, t)\right] d x d t \\
\quad+\int_{-\infty}^{\infty} \phi(x, 0) \rho_{0}(x) d x=0, \\
\iint_{\Pi_{T}}\left[\phi_{t} \cdot(\rho u)(x, t)+\phi_{x} \cdot\left(\rho u^{2}+p\right)(x, t)+\phi \cdot V(\rho(x, t), u(x, t), x, t)\right] d x d t \\
\quad+\int_{-\infty}^{\infty} \phi(x, 0) \rho_{0}(x) u_{0}(x) d x=0 .
\end{gathered}
$$

This completes the proof of the theorem.

From Theorem 5, Theorem 6 and the results of [9], we immediately obtain Theorem 2 (Sect. 1).

Acknowledgements. The authors are grateful to Professors P. D. Lax and J. Glimm for their enthusiastic encouragement. They are indebted to Professor R. J. DiPerna for valuable remarks.

\section{References}

1. Zhang Tong, Guo Yu-Fa: A class of initial-value problem for systems of aerodynamics equations. Acta Math. Sinica 15, 386-396 (1965)

2. Nishida, T.: Global solution for an initial-boundary-value problem of a quasilinear hyperbolic system. Proc. Jpn. Acad. 44, 642-646 (1968)

3. Bahkvarov, N.: On the existence of regular solutions in the large for quasilinear hyperbolic systems. Ah. Vychisl. Mat. Fiz. 10, 969-980 (1970) 
4. Nishida, T., Smoller, J.: Solutions in the large for some nonlinear hyperbolic conservations. Commun. Pure Appl. Math. 26, 183-200 (1973)

5. Ding Shia-shi, Chang-Tung, Wang Ching-Hua, Hsiao-Ling, Li Tsai-Chang: A study of the global solutions for quasilinear hyperbolic systems of conservation laws. Scientica Sinica 16, 317-335 (1973)

6. Lin Longwei: A study of the global solutions for system of gas dynamics. Acta Scien. Nat. Jilin Univ. 1, 96-106 (1978)

7. DiPerna, R. J.: Convergence of the viscosity method for isentropic gas dynamics. Commun. Math. Phys. 91, 1-30 (1983)

8. Ding Xiaxi, Chen Gui-Qiang, Luo Peizhu: Convergence of the Lax-Friedrichs scheme for isentropic gas dynamics (I), (II). Acta Math. Sci. 5, 483-500, 501-540 (1985)

9. Chen Gui-Qiang: Convergence of the Lax-Friedrichs scheme for isentropic gas dynamics (III), ibid. 6, 75-120 (1986)

10. Ding Xiaxi, Chen Gui-Qiang, Luo Peizhu: A supplement to the papers "Convergence of the Lax-Friedrichs scheme for isentropic gas dynamics (II)-(III)", ibid. 7, (1987)

11. Glimm, J.: Solutions in the large for nonlinear hyperbolic systems of equations. Commun. Pure Appl. Math. 18, 697-715 (1965)

12. Tartar, L.: Une nouvelle methode de resolution d'equations aux derivees partielles nonlineaire. Lecture Notes in Mathematics, vol. 665, pp. 228-241. Berlin, Heidelberg, New York: Springer 1977

13. Tartar, L.: Compensated compactness and applications to partial differential equations. In: Research notes in mathematics, nonlinear analysis and mechanics. Heriot-Watt Symposium, Vol. 4, (ed.) Knops, R. J. London: Pitman Press 1979

14. Tartar, L.: The compensated compactness method applied to systems of conservation laws. In: Systems of nonlinear partial differential equations, NATO ASI Series, Ball, J. M. (ed.). Darhecht: D. Reidel 1983

15. Murat, F.: Compacité par compensation. Ann. Scuola Norm. Sup. Pisa Sci. Fis. Mat. 5, 489-507 (1978)

16. Murat, F.: Compacité par compsensation: condition nécessaire et suffisante de continuité faible sous une hypothese de rang constant. Ann. Scuola Norm. Sup. Pisa 8, 69-102 (1981)

17. Murat, F.: L'injection du cone positif de $H^{-1}$ dans $W^{-1, q}$ est compact pour tout $q>0$. J. Math. Pures Appl. 6, 309-322 (1981)

18. Dacorogna, B.: Weak continuity and weak lower semicontinuity of nonlinear functionals. Lecture Notes in Mathematics, vol. 922, pp. 1-20. Berlin, Heidelberg, New York: 1982

19. Lax, P.: Weak solutions of nonlinear hyperbolic equations and their numerical computation. Commun. Pure Appl. Math. 7, 159-193 (1954)

20. Godunov, S. K.: A difference method for numerical calculation of discontinuous solutions of the equation of hydrodynamics. Mat. Sb. 47, 89, 271-306 (1959)

21. DiPerna, R. J.: Convergence of approximate solutions to conservation laws. Arch. Rat. Mech. Anal. 82, 27-70 (1983)

22. DiPerna, R. J.: Compensated compactness and general systems of conservation laws, preprint

23. Ball, J. M.: Convexity conditions and existence theorem in nonlinear elasticity. Arch. Rat. Mech. Anal. 63, 337-403 (1977)

24. Oleinik, O.: Discontinuous solutions of nonlinear differential equations. Usp. Mat. Nauk. (N.S.) 12, 3-73 (1957)

25. Conway, E., Smoller, J.: Global solutions of the Cauchy problem for quasilinear first-order equations in several space variables. Commun. Pure Appl. Math. 19, 95-105 (1966)

26. Kruzkov, S.: First-order quasilinear equations with several space variables. Mat. Sb. 123, 228-255 (1970)

27. Morawetz, C. S.: On a weak solution for a transonic flow problem. Commun. Pure Appl. Math. 38, 797-818 (1985)

28. Serre, D.: La compacite' par compensation pour les systems hyperboliques nonlineaires de deux equations a une dimension d'espace, preprint

29. Rascle, M.: Une resultat de "Compacité par compensation á coefficients variables", Application à l'elasticité nonlinear. C.R.A.S. 302, 311-314 (1986)

30. Roytburd, V., Slemrod, M.: An application of the method of compensated compactness to a problem 
in phase transitions. Proc. of the Symposium (to appear). Year on Material Instabilities and Continuum Mechanics. Oxford: Oxford University Press

31. Dafermos, C. M.: Solutions in $L^{\infty}$ for a conservation law with memory LCDS/CCs \# 87-5

32. Liu Tai-ping: Quasilinear hyperbolic systems. Commun. Math. Phys. 68, 141-172 (1979)

33. Ying Lung-an, Wang Ching-hua: Global solutions of the Cauchy problem for a nonhomogeneous quasilinear hyperbolic system. Commun. Pure Appl. Math. 33, 579-597 (1980)

34. Ying Lung-an, Wang Ching-hua: Solution in the large for nonhomogeneous quasilinear hyperbolic systems of equations. J. Math. Anal. 78, 440-454 (1980)

35. Dafermos, C. M., Hsiao, L.: Hyperbolic systems of balance law with inhomogeneity and dissipation. Indiana U. Math. J. (c) 31, 471-491 (1982)

36. Chueh, K., Conley, C., Smoller, J.: Positively invariant regions for systems of nonlinear diffusion equations. Indiana U. Math. J. 26, 373-392 (1977)

Communicated by A. Jaffe

Received July 19, 1988 